\title{
EVOLUTION OF SPINNING AND BRAIDING HELICITY FLUXES IN SOLAR ACTIVE REGION NOAA 10930
}

\author{
B. Ravindra ${ }^{1}$, Keiji Yoshimura ${ }^{2}$, and Sergio Dasso ${ }^{3,4}$ \\ ${ }^{1}$ Indian Institute of Astrophysics, Koramangala, Bangalore 560 034, India; ravindra@iiap.res.in \\ 2 Department of Physics, Montana State University Bozeman, MT 59717, USA; yosimura@ solar.physics.montana.edu \\ ${ }^{3}$ Instituto de Astronomía y Física del Espacio (CONICET-UBA), 1428 Buenos Aires, Argentina \\ ${ }^{4}$ Departamento de Física, Facultad de Ciencias Exactas y Naturales (UBA), 1428 Buenos Aires, Argentina; dasso@df.uba.ar \\ Received 2011 January 10; accepted 2011 August 19; published 2011 November 21
}

\begin{abstract}
The line-of-sight magnetograms from Solar Optical Telescope Narrowband Filter Imager observations of NOAA Active Region 10930 have been used to study the evolution of spinning and braiding helicities over a period of five days starting from 2006 December 9. The north $(\mathrm{N})$ polarity sunspot was the follower and the south (S) polarity sunspot was the leader. The N-polarity sunspot in the active region was rotating in the counterclockwise direction. The rate of rotation was small during the first two days of observations and it increased up to $8^{\circ} \mathrm{hr}^{-1}$ on the third day of the observations. On the fourth and fifth days it remained at $4^{\circ} \mathrm{hr}^{-1}$ with small undulations in its magnitude. The sunspot rotated about $260^{\circ}$ in the last three days. The S-polarity sunspot did not complete more than $20^{\circ}$ in five days. However, it changed its direction of rotation five times over a period of five days and injected both the positive and negative type of spin helicity fluxes into the corona. Through the five days, both the positive and negative sunspot regions injected equal amounts of spin helicity. The total injected helicity is predominantly negative in sign. However, the sign of the spin and braiding helicity fluxes computed over all the regions were reversed from negative to positive five times during the five-day period of observations. The reversal in spinning helicity flux was found before the onset of the X3.4-class flare, too. Though, the rotating sunspot has been observed in this active region, the braiding helicity has contributed more to the total accumulated helicity than the spinning helicity. The accumulated helicity is in excess of $-7 \times 10^{43} \mathrm{Mx}^{2}$ over a period of five days. Before the X3.4-class flare that occurred on 2006 December 13, the rotation speed and spin helicity flux increased in the S-polarity sunspot. Before the flare, the total injected helicity was larger than $-6 \times 10^{43} \mathrm{Mx}^{2}$. The observed reversal in the sign of spinning and braiding helicity fluxes could be the signature of the emergence of a twisted flux tube, which acquires the writhe of an opposite sign. The magnetic cloud associated with the ejected mass has carried about $-7 \times 10^{41} \mathrm{Mx}^{2}$ of helicity. A time integration of helicity flux of about $1.2 \mathrm{hr}$ integrated backward in time of the observation of the coronal mass ejection is sufficient for this event.
\end{abstract}

Key words: Sun: coronal mass ejections (CMEs) - Sun: evolution - Sun: flares - sunspots

\section{INTRODUCTION}

Coronal mass ejection (CME) is an energetic and spectacular event occurring on the Sun. This is also a driver for space weather and interplanetary magnetic disturbances. The ejected flux rope observed in the interplanetary media is called a magnetic cloud (MC) and is also known as an interplanetary CME. The MCs carry the magnetic field and particles along with them, leaving the footpoints anchored to the Sun. The observed MCs take the shape of a helical structure due to the rotation across the cloud (Klein \& Burlaga 1982). The observed magnetic fields in CMEs are assumed to originate from the active region, below the surface (Low 1994; Fan \& Gibson 2004). Hence, it is believed that the amount of helicity injected into the corona should be carried away by the ejected flux rope.

During the declining phase of solar cycle 23, on 2006 December 13 the largest halo CME was observed on the Sun. This CME occurred on the Sun followed by the X3.4class flare, coronal dimming, and EIT waves. The event produced interplanetary shocks, solar energetic particle events, and MCs. This event was detected by the Large Angle and Spectroscopic Coronagraph (LASCO) and later the MCs went past the Wind spacecraft. The propagation of the CME and shock in the interplanetary medium is well studied by Liu et al. (2008). The source location of the CME coincides with the active region (AR) NOAA 10930.
AR 10930 has seen five solar rotations. In the first solar rotation the active region was given the number NOAA 10923. It appeared on 2006 November 8 on the east limb of the Sun. During that period it had only the main, large south polarity sunspot while the north polarity region was spread into plage areas. In this phase, the active region was quiet and no major flare was reported. In the next solar rotation on 2006 December 8, the south polarity was still large in size, and the north polarity sunspot was emerging in the southern portion of it, which continued until December 14. The active region during this rotation was called NOAA 10930. This active region produced four $\mathrm{X}$-class and five $\mathrm{M}$-class flares. In the next two successive solar rotations, the south polarity region remained as it is (AR 10935 and AR 10941) and the north polarity region disappeared. In the fifth solar rotation only a small, spread-out region was remaining. More details about its evolution over four solar rotations can be found in Tiwari et al. (2008) and also at http://www.solarmonitor.org/.

Many researchers have studied the NOAA 10923 region during its second rotation on the Sun. During this time the active region was named AR 10930. It was also the period when the large amount of flux was emerging. The active region has produced several large flares and CMEs that were well observed by the Solar and Heliospheric Observatory, the Transition Region and Coronal Explorer (TRACE), Hinode, and RHESSI spacecraft at various wavelengths. Many results are published on the X3.4-class flare event alone. 
Most of the observational studies of this active region are concentrated on the changes in the parameters before and after the X3.4-class flare. Some of them are listed here. Kubo et al. (2007) observed that the transverse field has changed after the flare in the active region. Jing et al. (2008a) measured the distribution of the magnetic reconnection rate in the flare ribbons. Jing et al. (2008b) also found that after the X3.4-class flare there was an increase in the magnetic shear below the height of $8 \mathrm{Mm}$ and a decrease in the shear from 8 to $70 \mathrm{Mm}$. Magara (2009) observed the decrease in the shear before the onset of the X3.4-class flare. Yan et al. (2009) observed that the X3.4class flare occurred after the rapid counterclockwise rotation of the sunspot. Ravindra et al. (2011) found one complete winding in the active region one day before the flare. Abramenko et al. (2008) observed that when the kinetic vorticity of the plasma flows reached its maximum, the intermittency in the photospheric magnetic field also peaked, with a delay of $4 \mathrm{hr}$. The X3.4-class flare was observed 1.3 days after the peak of the photospheric intermittency. Schrijver et al. (2008) found a drastic reduction in the current after the flare in high arching arcade. Also, the long current carrying the fibril that was low lying near the surface disappeared after the flare.

There have been several studies on the release of free energy during the X3.4-class flare. With the Solar Optical Telescope (SOT) Spectropolarimeter (SP) data and using the NLFFF algorithm, Schrijver et al. (2008) reported that there is a reduction of about $3 \times 10^{32}$ erg of free energy after the flare compared to the pre-flare energy. Guo et al. (2008) estimated about a $2.4 \times 10^{31}$ erg decrease in energy after the flare. On the other hand, Jing et al. (2010) observed a continuous increase in the free energy before and after the X3.4-class flare. By applying the magnetic virial theorem on the pre-processed vector magnetograms, Ravindra \& Howard (2010) found that there is a decrease in the magnetic free energy of about $3.1 \times$ $10^{32}$ erg after the flare compared to the pre-flare energy. They concluded that half of the released energy is taken away by the $\mathrm{CME}$ and the rest is utilized by the radiative energy loss, particle acceleration, plasma, and magnetic field reorientation.

In many occasions, the twist in the active region is studied and looked at for its change during energetic events. Magnetic helicity is considered as a parameter that represents the twist and twine in the magnetic flux tubes. A lot of work has been done toward the injection of helicity into the corona measured by following the photospheric magnetic footpoints (Zhang et al. 2008; Park et al. 2010) in AR 10930. Zhang et al. (2008) reported a jump in the rate of helicity from negative to positive during the onset of the X3.4-class flare. A similar result was obtained by Park et al. (2010). They estimated that $-4.3 \times 10^{43} \mathrm{Mx}^{2}$ of helicity flux was injected into the corona before the X3.4class flare. The region NOAA 10930 is an interesting emerging active region and during its disk passage there was no other active region visible on the Sun. Hence, we can be sure that the observed changes in the helicity are not caused by the exchange of the helicity between the neighboring active regions (Yang et al. 2009). Longcope et al. (2007) have found that the helicity flux measured with the low-resolution Michelson Doppler Imager (MDI) magnetogram underestimates by a factor of two compared to the high-resolution MDI magnetograms. The SOT/Narrowband Filter Imager (NFI) has made the Stokes $V / I$ maps of this active region starting from 2006 December 9-14. These magnetograms provide a unique opportunity to estimate the injected helicity flux into the corona using very high spatial resolution magnetograms. While the AR 10930 region was emerging, sunspots in the active region had rotational and braiding motions around one another. It is interesting to see which of these motions contributed more to the total injected helicity flux: Is it the spinning motion or the braiding motion of the footpoints? Also, it is interesting to compare the net injected helicity and the ejected helicity.

In this paper, we study the evolution of spinning and braiding helicities in AR NOAA 10930 and examine which one has contributed more to the total helicity flux. It is interesting to study the sign and magnitude of the spin and braiding helicity fluxes as it can provide information about the coronal helicity due to the sub-photosphere dynamics while the flux tube is emerging. We also look for the changes (if any) in the helicity flux before, during, and after the flare. Finally, we examine how much helicity the active region accumulated over a period of five days of observations and also how much the halo CME (on 2006 December 13) was carried away with it. This paper is structured as follows. In the next section, we describe the details about the Hinode imaging line-of-sight magnetogram and vector magnetogram data. A description of how the Stokes $V$-signal from the imaging magnetograph is converted into useful magnetograms is given in brief. We also describe briefly the method adopted to estimate the spin and braiding helicity flux. In Section 3, we give detailed results on the comparison of the evolution of the braiding and spinning helicity flux. We also compute how much the sunspot rotated during the period of its evolution. We then compare the injected helicity with the helicity carried away by the halo CME observed on December 13 . In the last section, we discuss the results and compare them with the findings of others.

\section{OBSERVATIONAL DATA AND METHODS OF ANALYSIS}

The space-based solar telescope, SOT/SP (Tsuneta et al. 2008; Suematsu et al. 2008; Ichimoto et al. 2008) on board the Hinode (Kosugi et al. 2007) satellite makes polarization maps of the active region by spatial scanning. The spectropolarimeter obtains the Stokes $I, Q, U$, and $V$ spectra in Fe I $6301.5 \AA$ and $6302.5 \AA$ lines. We obtained three components of the vector magnetic field measurements of the AR 10930 from the level-2 data archive (http://sot.lmsal.com/data/sot/level2dd). These vector field data have been used to calibrate the Stokes $V$-signal obtained from the narrowband filtergrams (NFI) on SOT.

In order to compute the helicity fluxes in AR 10930 we have obtained the data from SOT/NFI. The Stokes $I$ and $V$ image data have been obtained in the $6302 \AA$ line. The Stokes signal has been obtained using NFI, which was positioned at $120 \mathrm{m \AA}$ in the blue wing of the spectral line. We obtained Stokes $I$ and $V$ maps over a period of five days starting from 2006 December 9 at 00:00 UT until 2006 December 14 at 02:30 UT and collected about 3000 Stokes $I$ and $V$ maps. The obtained data are almost uniform in time cadence (about 2 minutes) with some small data gaps on December 14. The SOT/NFI data have a pixel resolution of 0". 16 in the spatial $x$ - and $y$-directions. The Stokes $I$ and $V$ data sets are corrected for dark and bias using the standard solar software pipeline. The inverted magnetograms of SOT/SP are used to convert the Stokes $V$ signal (of SOT/NFI) into useful line-of-sight magnetograms as has been done by Chae et al. (2007). The difference between their method and ours is that we have used the inverted SP magnetograms instead of the center-of-gravity method to obtain the line-ofsight magnetograms from SOT/SP. More details about the data 
reduction and conversion can be found in Chae et al. (2007). While converting the SOT/NFI data to useful magnetograms we have taken the pixel resolution of the SOT/SP into account. We used the line-of-sight magnetograms from SOT/SP starting from December 9 to 14 (whenever it was available) to correct the line-of-sight magnetograms of SOT/NFI. We then constructed a data cube of magnetograms as well as intensity images by extracting the rectangular box enclosing the AR 10930 within it. In the time sequence, four magnetograms have been averaged to reduce the noise level and the central magnetogram time is taken as a reference time. We corrected the magnetograms for the viewing angle by assuming the magnetic field is radial at the measurement height. The line-of-sight measurement is divided by the cosine of the angle from the disk center to convert to a vertical field.

The calibrated Stokes $I$ images have been filtered to remove the acoustic waves present in the images. We used a subsonic filter with a phase velocity cutoff value of $5 \mathrm{~km} \mathrm{~s}^{-1}$ to remove the acoustic oscillations as has been done in Title et al. (1989). These filtered intensity images have been used to track the features in and around the active region.

The transverse velocity fields have been computed using the local correlation tracking (LCT; November \& Simon 1988) technique. The LCT has been applied to the intensity images rather than the magnetograms. After several experimentations we choose the time interval between the pair of intensity images as 4 minutes and the size of the Gaussian apodizing window as $4 "$. We averaged two such velocity images to reduce the noise in the velocity measurements, and also the cadence is the same as the four averaged magnetograms. The final magnetograms and velocitygrams have a uniform time cadence of 8 minutes.

\subsection{Magnetic Helicity Flux}

In the photosphere, the helicity flux changes due to the emergence/submergence of the magnetic field lines and/or due to the shearing motion of the footpoints. Assuming ideal MHD flow at the boundary, Berger (1999) derived the equation for the time derivative of the helicity, which is given as

$$
\frac{d H}{d t}=-2 \oint_{s}\left[\left(\mathbf{A}_{p} \cdot \mathbf{v}_{t}\right) B_{n}-\left(\mathbf{A}_{p} \cdot \mathbf{B}_{t}\right) \cdot v_{n}\right] \cdot d S,
$$

where $B_{n}$ and $\mathbf{B}_{\mathbf{t}}$ are the normal and transverse components of the magnetic field measured at one level. $v_{n}$ and $\mathbf{v}_{\mathbf{t}}$ are the normal and transverse components of the velocity of the magnetic footpoints. The first term on the right-hand side of the equation represents the change in helicity due to the shearing motion of the footpoint and the second term is due to the flux emergence/submergence. Démoulin \& Berger (2003) have shown that if there is any vertical flow $\left(v_{z}\right)$ across the boundary, then the horizontal component of the velocity can be combined with it and the resulting velocity (u) is given by

$$
\mathbf{u}=\mathbf{v}_{h}-\frac{v_{z}}{B_{z}} \mathbf{B}_{\mathbf{h}}
$$

Utilizing this in Equation (1) reduces the equation to

$$
\frac{d H}{d t}=-2 \int_{z=0}\left(\mathbf{u} \cdot \mathbf{A}_{p}\right) B_{z} d S,
$$

where $\mathbf{A}_{p}$ is the vector potential for the potential magnetic field. Given this equation along with magnetic and velocity fields, one can derive the rate of injected helicity at the photospheric level (e.g., Chae et al. 2001; Park et al. 2010).

Brown et al. (2003) have measured the rotation speed of the rotating sunspot in seven active regions, which are at different stages of evolution. Longcope et al. (2007) have studied the spin helicity fluxes in the same active regions and derived the rate of rotation of sunspots that are studied by Brown et al. (2003). In this comparison of the rate of rotation, Longcope et al. (2007) have used the line-of-sight magnetograms from MDI (Scherrer et al. 1995). Longcope et al. (2007) have reported that the rate of rotation of a sunspot obtained by using the low-resolution magnetograms underestimates by a factor of two compared to the rate of rotation of the same sunspot reported by Brown et al. (2003). On the other hand, the results closely match with Brown et al. (2003) when Longcope et al. (2007) used a high-resolution MDI magnetogram to estimate the rotation rate of sunspots. Since the rotation rate is directly related to the spin helicity flux, they concluded that there is an underestimation of the spin helicity flux if one uses the low-resolution magnetograms. Because of this, we planned to use the time sequence of highresolution magnetograms from SOT/NFI.

\subsection{Partitioning the Active Region Magnetic Fields}

In order to estimate the spinning and braiding helicity fluxes in the active region, we partitioned the calibrated magnetograms into a set of unipolar regions (Barnes et al. 2005; Longcope et al. 2007). The method of Longcope et al. (2007) is adopted here to perform such partitions. A gradient-based tessellation algorithm has been used here to perform the partitioning of the regions (Hagenaar 1999; Barnes et al. 2005; Longcope et al. 2007). A threshold of $50 \mathrm{G}$ is chosen for the partitioning algorithm to group the pixels. Smaller regions are merged with larger ones by choosing the saddle point merging. A saddle point value of $1500 \mathrm{G}$ has been chosen, below which it separates the regions. The resulting partition encloses the large sunspots as a whole and will be discussed in Section 3. In other words, the method assigns a unique number for the pixels available in one partition. The active region evolved over a period of five days. To track the regions and to minimize the new partitions we advect the partitions from the last time step. This method is more stable when long time series and emerging flux regions are considered. More details about the partitioning algorithm and its limitations can be found in Longcope et al. (2007).

\subsection{Spinning and Braiding Helicity Fluxes}

The rate of change of magnetic helicity can be estimated using Equation (3). Longcope et al. (2007) have decomposed the helicity flux into two terms by restricting the region of integration as

$$
\begin{aligned}
\frac{d H}{d t}= & -2 \sum_{a} \int_{\mathcal{R}_{a}}\left[\mathbf{A}_{P}^{a}(\mathbf{x}) \cdot \mathbf{u}(\mathbf{x})\right] B_{z}(\mathbf{x}) d^{2} x \\
& -\frac{1}{\pi} \sum_{a} \sum_{b \neq a} \int_{\mathcal{R}_{a}} \int_{\mathcal{R}_{b}} \frac{\hat{\mathbf{z}} \times\left(\mathbf{x}-\mathbf{x}^{\prime}\right) \cdot \mathbf{u}(\mathbf{x})}{\left|\mathbf{x}-\mathbf{x}^{\prime}\right|^{2}} B_{z}\left(\mathbf{x}^{\prime}\right) \\
& \times B_{z}(\mathbf{x}) d^{2} x^{\prime} d^{2} x
\end{aligned}
$$

The first term represents the spinning helicity flux obtained from individual partitions and the second term represents the braiding helicity flux. The vector potential $A_{P}$ has been decomposed into the sum of vector potential from each of the 


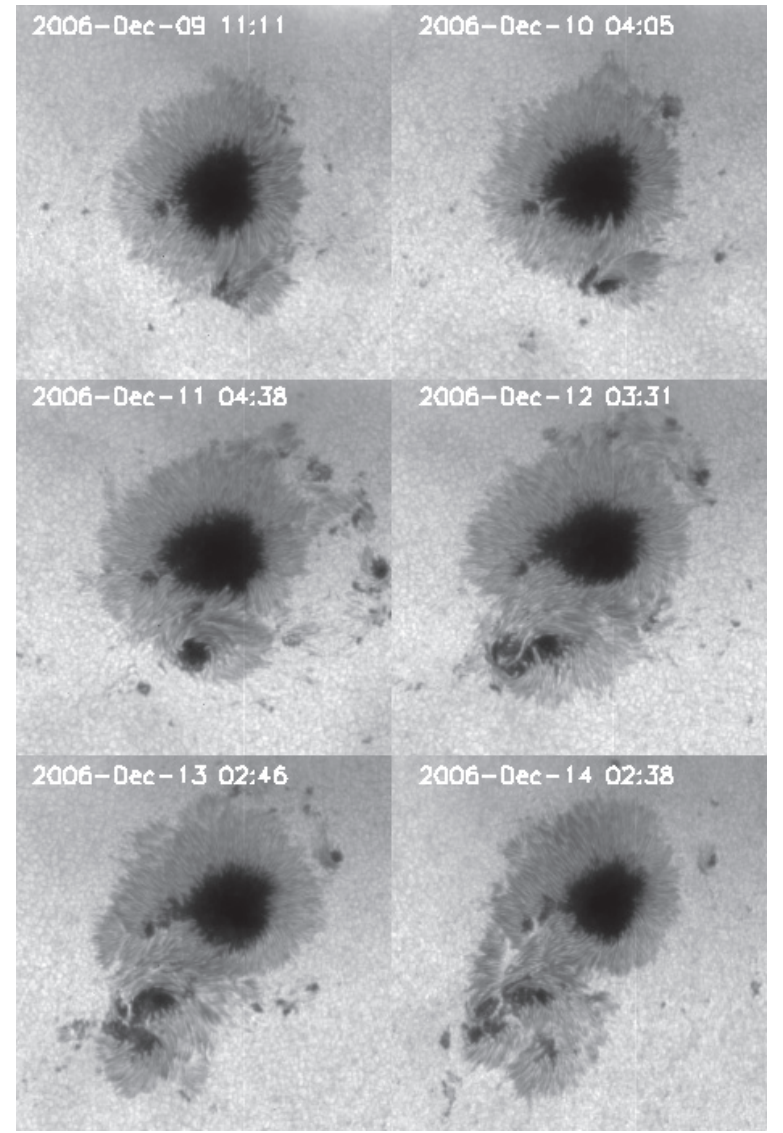

Figure 1. Time sequence of $I$-maps of NOAA AR 10930 taken in the blue wing of the 6302 Å line.

partitions by restricting the region of integration as

$$
\mathbf{A}_{P}^{a}(\mathbf{x})=\frac{1}{2 \pi} \int_{\mathcal{R}_{a}} \frac{\hat{\mathbf{z}} \times\left(\mathbf{x}-\mathbf{x}^{\prime}\right)}{\left|\mathbf{x}-\mathbf{x}^{\prime}\right|^{2}} B_{z}\left(\mathbf{x}^{\prime}\right) d^{2} x^{\prime} .
$$

Once the rate of spinning helicity flux is known, it is easy to estimate the average angular rotation rate and is given by

$$
\bar{\omega}_{a} \equiv \frac{4 \pi}{\Phi_{a}^{2}} \int_{\mathcal{R}_{a}}\left[\mathbf{A}_{P}^{a} \cdot\left(\mathbf{u}-\overline{\mathbf{u}}_{a}\right)\right] B_{z} d^{2} x .
$$

More details about the test results on the rate of spinning and braiding helicity fluxes and its application to the active regions can be found in Longcope et al. (2007).

\section{RESULTS}

The active region NOAA 10930 emerged during the declining phase of solar cycle 23. The active region appeared on the east limb of the Sun on 2006 December 5 in the southern hemisphere at a latitude of $5^{\circ}$. The active region had two polarities when it appeared on the east limb and they were almost aligned perpendicular to the east-west direction. It was still visible when it crossed the west limb on December 17. It produced an X3.4-class flare in the scale of the GOES instrument on 2006 December 13 beginning at 02:14 UT and ending at 02:57 UT. Figure 1 shows the time sequence of intensity images displayed for a period of six days. From Figure 1 and from the long time sequence of observations it appeared that the large sunspot is more stable. However, during our observation another sunspot was emerging in the southern portion of the main sunspot. It appeared to be rotating along its emergence (see also, Min \& Chae 2009). On December 12, a few light bridges appeared on this sunspot and later, on December 13, the sunspot split into two individual spots. On December 14, there were more than four spots present in the active region. Apart from rotation there was translational motion of the emerging sunspot. Hence, the boundary between the two sunspots was highly sheared (Ravindra et al. 2011).

\subsection{The Emerging Flux Region}

Figure 2 shows the time sequence of images of a line-ofsight magnetic field in NOAA AR 10930. It also shows the partitioning of the magnetic fields into unipolar regions. In this active region there are two large partitions (P01 and N01) and many smaller ones. The partitioning algorithm captured two large sunspots as an individual entity. As the sunspots grew, the two partition regions also enlarged in size while encapsulating the whole sunspot into it. A unique number is assigned to each partition. If the new region emerges, it gets a new number. However, large regions (P01 and N01) existed throughout our observational period and maintained their unique number.

Figure 3 (top) shows the magnetic flux evolution in the P01 and N01 regions over a period of five days. In the plot, the positive and negative flux evolutions are shown separately. In the beginning, the fluxes in P01 and N01 are almost constant and increased approximately linearly from December 10 at 20 UT until the end of the observations. There is a large flux imbalance in both partitions. The rates of flux emergence are $0.11 \times$ $10^{21} \mathrm{Mx} \mathrm{hr}^{-1}$ in P01 and $0.15 \times 10^{21} \mathrm{Mx} \mathrm{hr}^{-1}$ in N01. It is clear from Figure 2 that there is a large change in the area and shape of P01 and N01 over a period of five days. The different rate of flux emergence in $\mathrm{P} 01$ and N01 could be due to some portions of the magnetic flux emerging into the outside of the partitions. The dashed vertical line in the plot represents the onset time of the X3.4-class flare. The flux in the north polarity region was increasing rapidly until the onset of the X3.4-class flare and then afterward it increases at a slower rate.

When the active region emerges from the deep interior into the photosphere, the distance between the two polarities increases (Pevtsov et al. 2003). Figure 3 (middle) shows the plot of distance between P01 and N01 as a function of time. When the flux is constant from December 9-11, the distance between P01 and N01 increased slightly. However, when the flux started to increase after December 11, the distance between the two polarities increased at a rate of $63 \mathrm{~m} \mathrm{~s}^{-1}$ until December 13, and after that there is a small decrease in the separation between P01 and N01. The decrease in the separation starts just before the onset time of the X3.4-class flare. Another characteristic of the emerging flux region is the tilt. The tilt has been estimated as the angle between the line joining the flux-weighted centroids of P01 and N01 with respect to the equator. The tilt is measured in the counterclockwise direction and it increases from west to east in the southern hemisphere. Figure 3 (bottom) shows the change in the tilt between the two polarities with respect to the equator. Once again, when the flux is constant in P01 and N01, the tilt was almost constant until December 10. However, the tilt decreased continuously after December 10 until the onset of the X3.4-class flare. The rise in flux, increase in the distance between the two polarities, and decrease in the tilt all suggest that a new flux region is emerging into the visible layer of the Sun in AR 10930. 



Figure 2. Time sequence of images of line-of-sight magnetograms with the partitions. The $\mathrm{P}$ and $\mathrm{N}$ correspond to positive (north) and negative (south) polarity regions. The regions P01 and N01 are the large single partitions and they maintained their unique number throughout the observations. 


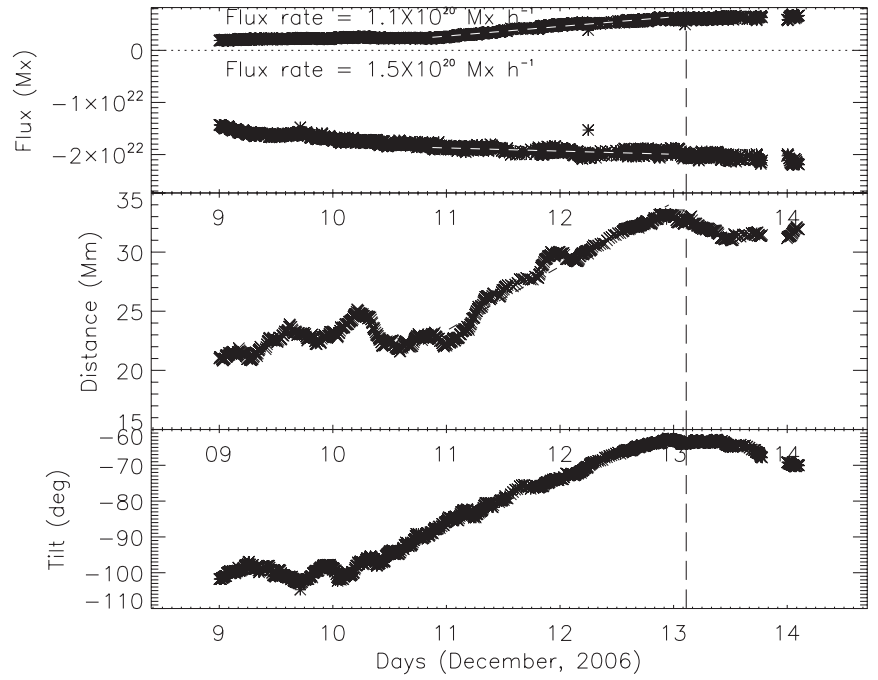

Figure 3. Top: plot of temporal evolution of magnetic flux in the P01 and N01 regions is shown for a period of five days. Middle: plot of separation between P01 and N01 polarities as a function of time. Bottom: plot of temporal evolution of the tilt angle between the line joining the P01 and N01 with the equator. The major tick marks in the $x$-axis correspond to days in 2006 December. The dashed vertical line represents the onset time of the X3.4-class flare.

\subsection{The Velocity Field in the Active Region}

In order to see the horizontal velocity patterns over the active region, we computed the velocities using the intensity images. The LCT technique has been adopted to track the features. Figure 4 shows the horizontal velocity vectors overlaid on the corresponding magnetograms. In the map, it is seen that there is inward flow as well as outward flow in the penumbra of the main large sunspot (N01) as seen in the past (Ravindra et al. 2004; Ravindra 2006). Apart from these, in the N-polarity sunspot (P01), the velocity patterns show the rotational motion. In order to see how the rotational pattern changes in the $\mathrm{N}$-polarity region with time, we have overlaid the computed horizontal velocity vectors subtracted by the mean velocity $(\mathbf{u}-\overline{\mathbf{u}})$ on the N-polarity region (Figure 5). The rotational pattern appeared clearly in the N-polarity region on 2006 December 11 and it was still present on 2006 December 14, during which time the north polarity sunspot split into several smaller units with separate umbra.

\subsection{The Rate of Rotation of the Sunspot and Injected Spin Helicity}

In order to examine how fast the $\mathrm{P} 01$ polarity region rotated, we used Equation (6) to estimate the rate of rotation. Figure 6 (top left) shows the rotational rate for region P01 as a function of time. The rotational velocity is small with large scatter in their values on 2006 December 9 and 10. It started increasing in the later phase of 2006 December 10 and reached a maxima on midday of December 11. During that time the rate of rotation was about $8^{\circ} \mathrm{hr}^{-1}$. It then decreased to $4^{\circ} \mathrm{hr}^{-1}$ on December 12 , showing a pattern of oscillations. During this period, the rate of rotation increased up to $5^{\circ} \mathrm{hr}^{-1}$ and then decreased to $3^{\circ} \mathrm{hr}^{-1}$. This pattern appeared as a damped oscillation and its peak amplitude is observed to be decreasing with time. A few hours before the onset of the X3.4-class flare the rotation rate increased up to $5^{\circ} \mathrm{hr}^{-1}$ and later it decreased to $3^{\circ} \mathrm{hr}^{-1}$.

Figure 6 (top right) shows the rotation rate for N01 region. By appearance, N01 was large in size and has a large amount of magnetic flux. It rotated at a rate of $0.5 \mathrm{hr}^{-1}$ to $1^{\circ} \mathrm{hr}^{-1}$ during its evolution. From December 9-12, it is observed that the direction of rotation of N01 was exactly opposite to the direction of rotation of the P01 spot. From then onward, region N01 changed its direction of rotation twice until December 14, though P01 spot did not change its direction of rotation. Region N01 changed its sign of rate of rotation during 10 UT on December 11. Once again it changed its sign on December 12, reached up to $1^{\circ} \mathrm{hr}^{-1}$, and, during the onset of the X3.4-class flare, decreased suddenly to a low value.

Once the sunspot rotates, it injects the spin helicity flux into the corona. Figure 6 (bottom left) shows the rate of change of the spin helicity injected into the corona by region P01 and the bottom right plot is for region N01. Both regions injected opposite and unequal amounts of helicity during the first two days of observations. On December 11, region N01 started injecting the helicity flux with a positive sign and P01 injected helicity flux with a negative sign. On December 12, the N01 region injected a negative helicity flux until the onset of the X3.4-class flare. However, during the onset of the flare, region N01 changed its sign of injected helicity flux to positive. Region P01 injected negative helicity flux all along December 11-14, though there is an undulation in its rate of injection and a few hours before the onset of flare its magnitude started decreasing.

Figures 7(a) and (b) show the net spin helicity flux and total rotation angle for regions P01 (green line) and N01 (red line) separately. Region P01 rotated by about $200^{\circ}$ over a period of five days. However, on December 9 and 10, the rate of rotation velocity was low and sometimes negative with small oscillations in their amplitudes. This would have reduced the total value of the rotation. If we consider the rate of rotation from 2006 December 11 until the end of 2006 December 13, the P01 spot rotated about $260^{\circ}$ over a period of three days. The N01 spot rotated only about $20^{\circ}$ over the period of five days. Even though the $\mathrm{P} 01$ region rotated more than half a turn, the majority of spin helicity contribution originates from the N01 region. However, at the end of $120 \mathrm{hr}$ of observations both the polarities (P01 and N01) injected a similar amount of spin helicity in excess of $-1.5 \times 10^{43} \mathrm{Mx}^{2}$. Further, there is a decrease in the spin helicity of N01 starting from 10 UT on December 11 until 12 UT on December 12. This is mainly due to the injection of opposite spin helicity flux into the corona. Then, it increased until the onset time of the X3.4-class flare. After the onset of the flare it becomes almost flat in shape.

\subsection{Spinning, Braiding, and Net Helicities}

Figure 8(a) shows the rate of change of net spinning (in green) and braiding (in red) helicity fluxes obtained from all the partitions as a function of time. The rates of spinning and braiding helicity fluxes are almost similar in magnitude except during the first half of December 11 and the second half of December 12. In the first half of December 11, the braiding helicity flux dominated over the spin helicity flux and in the second half of December 12 it is the other way. There are periods from 10-18 UT on December 11, 6-14 on December 12, 2-4 also at 9 and 16 UT on December 13 during which there is a change in the sign of both the spin and braiding helicity fluxes. It was negative most of the time period and reversed its sign during those periods. It should be noted here that the change in the sign of spinning and braiding helicity fluxes coincides with the time of enhancement of the rate of rotational velocity. The dashdotted yellow line in Figure 8(a) represents the sum of braiding and spin helicity fluxes. The black curve represents the rate of change of helicity computed independently using Equation (3). The dark vertical line represents the beginning of the X3.4-class 

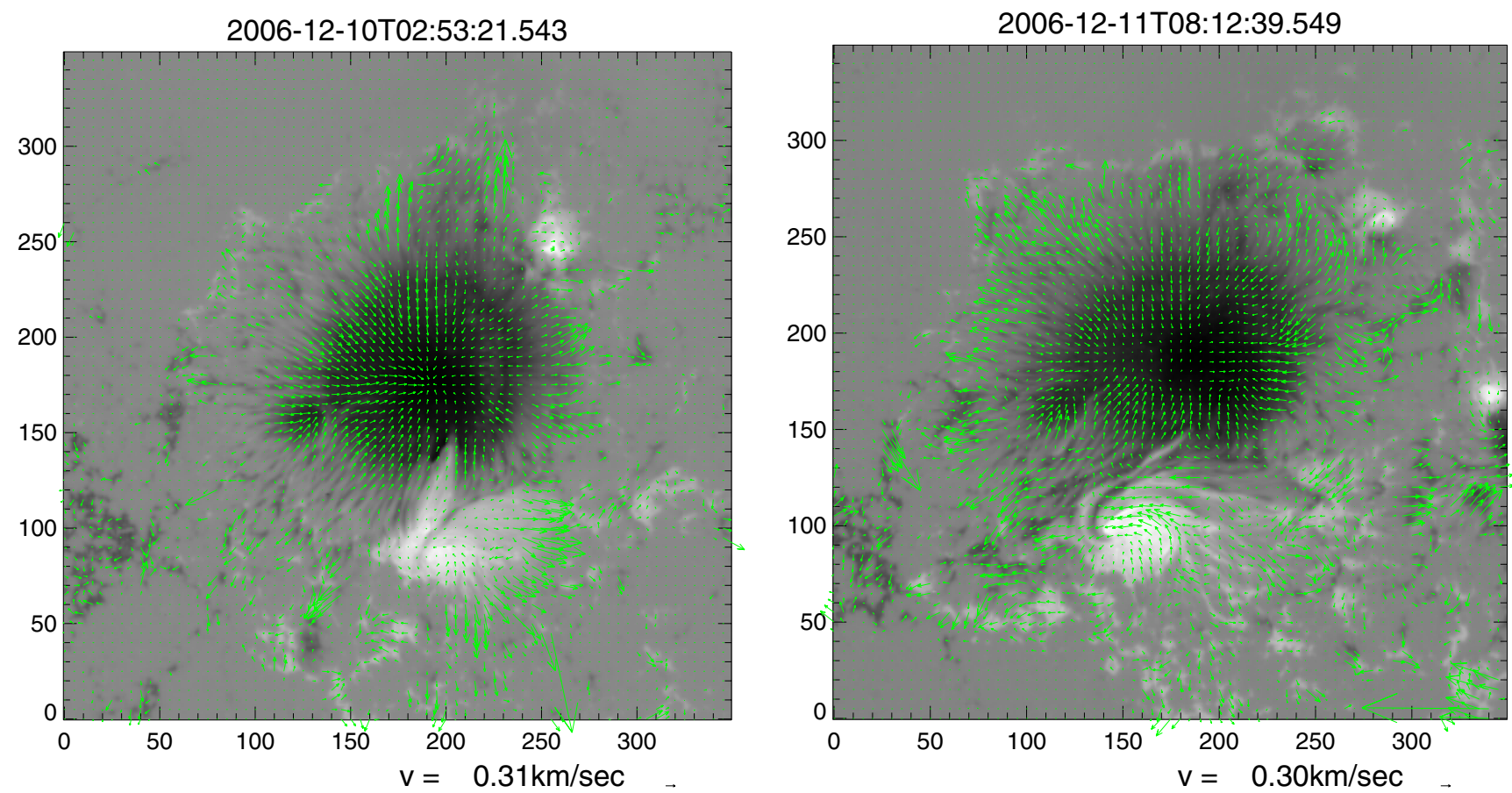

Figure 4. Horizontal velocity vectors overlaid upon the magnetogram image. Left and right images correspond to different days of observations.

flare that occurred on December 13, at 02:14 UT. Ten hours before the X3.4-class flare, the spin helicity flux increased in magnitude from $-0.5 \times 10^{42} \mathrm{Mx}^{2} \mathrm{hr}^{-1}$ to $-1.2 \times 10^{42} \mathrm{Mx}^{2} \mathrm{hr}^{-1}$. Just before the beginning of the X3.4-class flare the spinning helicity flux has changed its sign to positive. It remains positive for about $2 \mathrm{hr}$ and then again it reverses its sign. This could be due to the effect of the flare ribbons that appeared in the $V$ map and in those regions the calibration would not be correct. However, the flare ribbon persisted only for a few minutes and the change in the sign of spin helicity flux was present up to $1 \mathrm{hr}$ after the flare. Apart from these, we have averaged the magnetograms for about 8 minutes. Hence, the effect of flare ribbons on the helicity flux computation is negligible. We do not see any such long jump in the magnitude of the braiding helicity flux from mid-December 12 to mid-2006 December 13.

Figure 8(b) shows the total accumulated spinning (green line) and braiding (red line) and the sum of the spinning and braiding helicities (dotted yellow line) from all the partitions. From the plot it is clear that even though the P01 region rotated by $260^{\circ}$, more than half of the contribution to the total helicity originates from the braiding helicity term. The solid dark line in the plot represents the time integral rate of change of helicity estimated independently through the equation $\int(d H / d t) / d t$. A close matching of the time integral of the rate of change of helicity with the accumulated sum of spinning and braiding helicities (dash-dotted yellow line) suggests the correctness of the decomposition method to estimate the different helicity flux terms. Due to the reversal in the sign of the spinning and braiding helicity fluxes, there is a decrease in the magnitude of accumulated helicity many times throughout the observations (e.g., middle of December 10, middle of December 11, and middle of December 12). It should be noted here that the time-integrated spinning and braiding helicities are of the same sign throughout the observations and hence both add to the total accumulated helicity, though unequally. Before the onset of the X3.4-class flare the net accumulated helicity is larger than $-6 \times 10^{43} \mathrm{Mx}^{2}$. At the end of the fifth day of observations, the accumulated helicity was about $-7.5 \times 10^{43} \mathrm{Mx}^{2}$. This is a large amount of accumulated helicity for this moderately sized active region.

\subsection{Helicity in the Magnetic Cloud}

The Sun injects helicity during the emergence of large-scale (Nindos \& Zhang 2002) and small-scale (Welsch \& Longcope 2003) magnetic field structures and accumulates it in each hemisphere. The dissipation of magnetic helicity is so small that the Sun needs a mechanism to release it, and the eruption of CMEs is believed to be the main mechanism through which the Sun removes the excess of magnetic helicity (Low 1996).

From December 9-12 there were about 10 CMEs observed by the LASCO C2 coronagraph, which covers $2.0-6.0 R_{\odot}$ from the Sun (Brueckner et al. 1995). The largest (halo) CME happened on December 13 (at 2:54 UT). Before this event, the last CME was observed on December 12 at 21:47 UT. An MC that erupted from AR 10930 was observed at 1 astronomical unit (1 AU) on 2006 December 14. The detailed evolution of this event, from the Sun through the interplanetary medium, was studied by Liu et al. (2008). Harra et al. (2011) studied the details of its solar source using a velocity difference technique. The coronal counterpart for this MC was the largest halo CME that erupted on December 13, mentioned above, and it traveled near corona with a projected speed of $1774 \mathrm{~km} \mathrm{~s}^{-1}$, as observed by LASCO C2 (http://cdaw.gsfc.nasa.gov/CME_list/UNIVERSAL/2006_12/ univ2006_12.html).

Several authors have modeled the local magnetic configuration for MCs using Lundquist's model (Lundquist 1950), which considers a static and axially symmetric linear force-free magnetic configuration (see, e.g., Lepping et al. 1990; Lynch et al. 2003; Dasso et al. 2009). The magnetic structure for this model results in $\vec{B}=B_{0}\left[J_{1}(\alpha r) \phi+J_{0}(\alpha r) \mathbf{z}\right]$ (Lundquist 1950), with $r$ being the distance to the cloud axis, $B_{0}$ the magnetic field strength at the cloud axis, $\alpha / 2$ the twist of the field lines at the flux rope axis, and $J_{n}$ the Bessel function of the first kind of order $n$. 

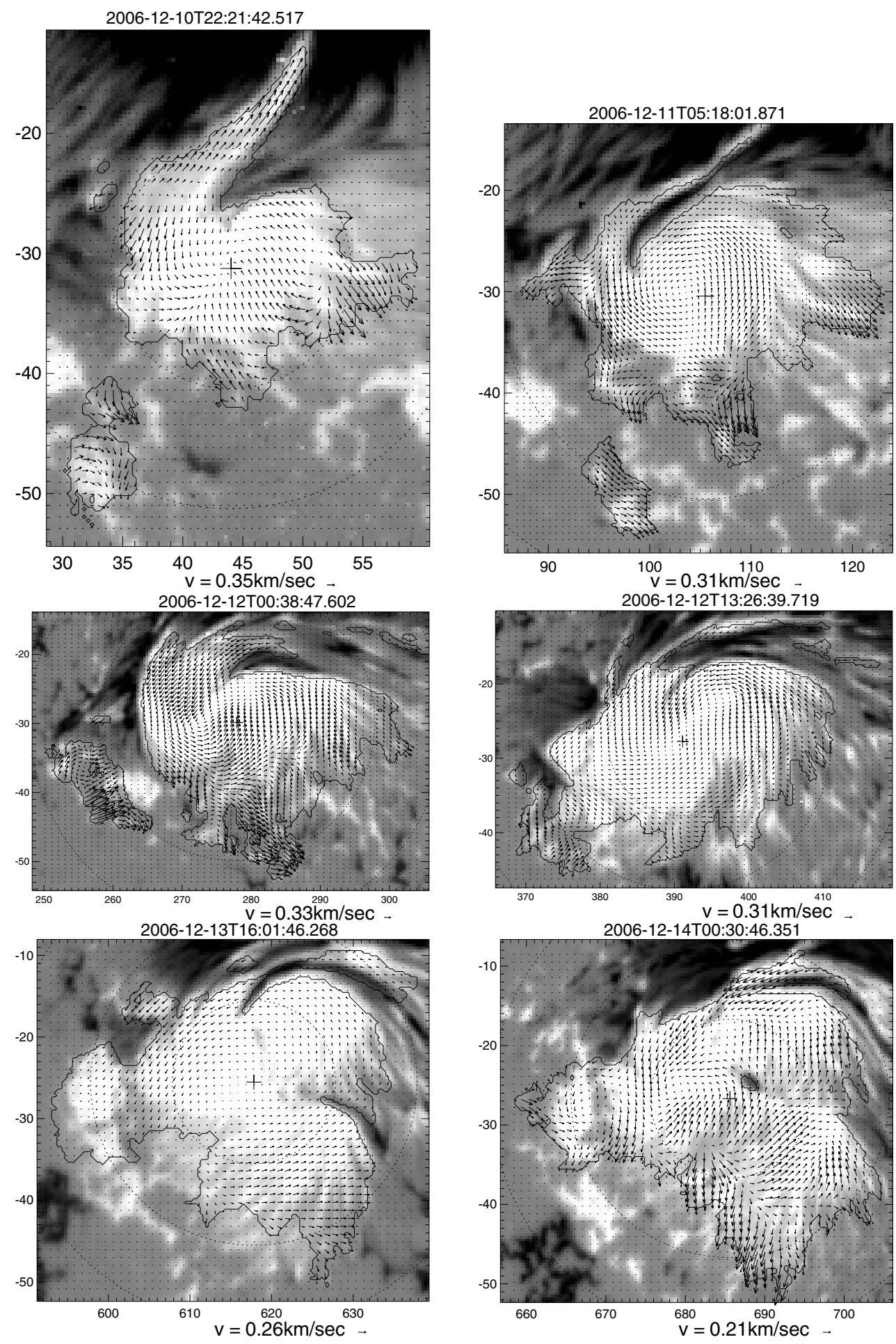

Figure 5. Horizontal velocity vectors overlaid upon the P01 region. The circles are drawn at a radius of $10^{\prime \prime}$ and the "+ " symbol represents the flux-weighted centroid for the selected partition (P01). The size of the arrow at the bottom of the figure represents the magnitude of the velocity.

From the analysis of in situ observations of magnetic and plasma properties inside the flux rope, it is possible to quantify the global content of magnetic helicity $\left(H_{M C}\right)$ in the MC (Dasso et al. 2003; Dasso 2009). Assuming Lundquist's model for the MC, $H_{\mathrm{MC}}$ can be computed as (Dasso et al. 2005)

$$
H_{\mathrm{MC}}=2 \pi\left(J_{0}^{2}+J_{1}^{2}-\frac{2 J_{0} J_{1}}{\alpha R}\right) \frac{L B_{0}^{2} R^{2}}{\alpha} \sim 0.7 B_{0} L R^{3}
$$

where $J_{0}$ and $J_{1}$ are the Bessel functions at $\alpha R$. $L$ and $R$ are the length and radius of the cylinder.

Quantitative studies comparing the release of magnetic helicity from the Sun, making a comparison of the amount of the coronal helicity before and after the eruption of the CME, have been carried out in some previous studies (see, e.g., the review by Démoulin 2008). In particular, a good agreement between the magnetic helicity contained in the MC and the release of helicity from the solar corona was found from a comparative study 

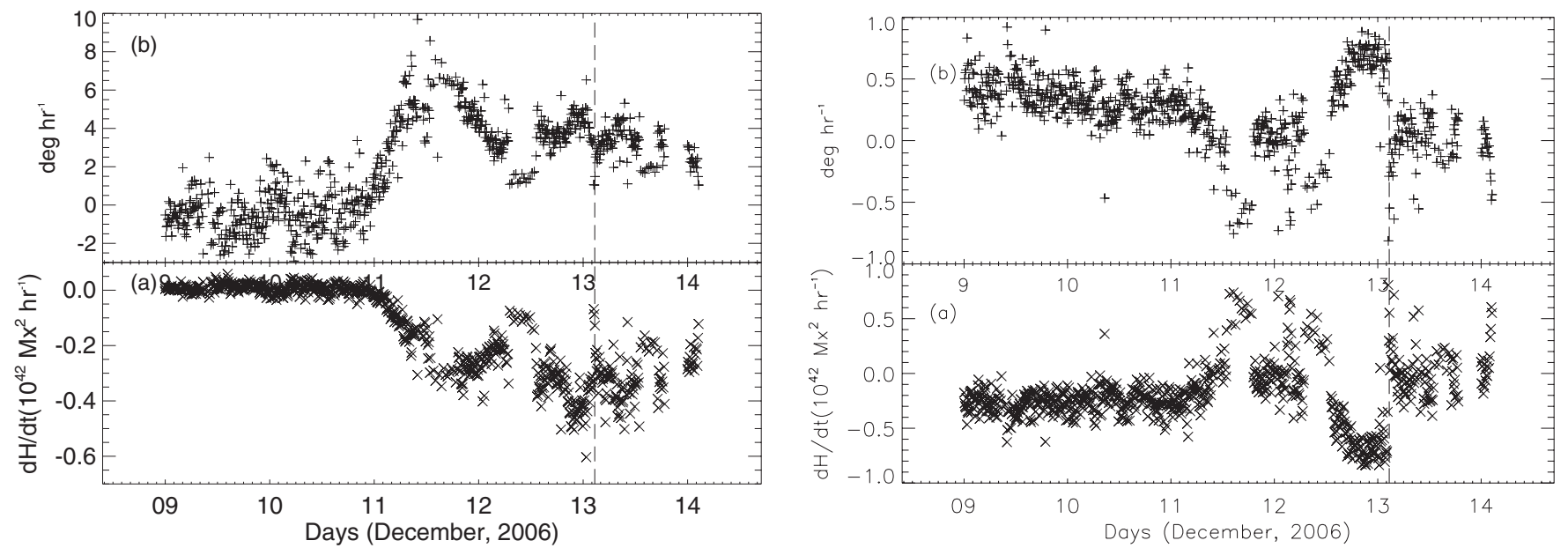

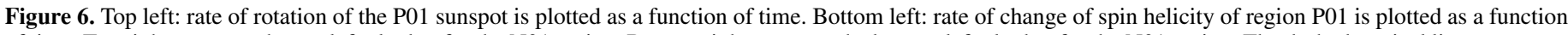

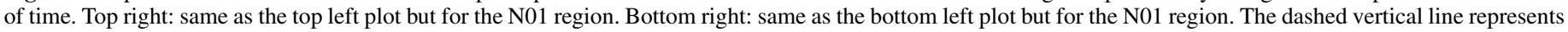
the onset time of the X3.4-class flare.

of MCs with very different sizes (Luoni et al. 2005; Mandrini et al. 2005).

The MC observed at $1 \mathrm{AU}$ on December 14 was modeled by Harra et al. (2011). By fitting the free parameters of Lundquist's model to the in situ observations of the Advanced Composition Explorer spacecraft, they found $B_{0}=18.8 \mathrm{nT}$ and $\alpha=-15.8 \mathrm{AU}^{-1}$. From the bulk proton velocity, the orientation of the flux rope, and the range of time of the observation of the event, a radial size of $R=0.06 \mathrm{AU}$ was obtained. On the other hand, Liu et al. (2008) reported the presence of bi-directional streaming of supra-thermal electrons while the cloud was observed at $1 \mathrm{AU}$, which are typically observed when the legs of the MC are anchored to the solar surface. Then, the length of the cylinder can be estimated as $L=2.4 \mathrm{AU}$. From these values and using Equation (7), we estimate the amount of the helicity in the cloud as $H_{\mathrm{MC}}=-7 \times 10^{41} \mathrm{Mx}^{2}$.

In order to compare the helicity carried away by the CME with the injected helicity, we integrated the rate of the injected helicity backward in time starting from the CME first observed by the $\mathrm{C} 2$ coronagraph. It was found that just $1.2 \mathrm{hr}$ of helicity injection was sufficient and during this time the active region injected about $-8.16 \times 10^{41} \mathrm{Mx}^{2}$ of helicity into the corona. It should be noted here that though the triggering of the flare/CME could have originated by the injection of opposite helicity flux, the helicity carried by the CME is negative in sign. This could be due to the fact that the CME carries the helicity, which is already accumulated in the corona.

\section{DISCUSSIONS}

Longcope et al. (2007) introduced a method for breaking up the helicity flux into spinning and braiding helicity fluxes by partitioning the magnetogram into unipolar regions. They applied this technique to low-resolution and high-resolution MDI magnetograms and found that the helicity flux estimated using the MDI low-resolution magnetograms underestimates the helicity flux by a factor of two. We studied the spinning and braiding helicity fluxes in the AR 10930 over a period of five days. We used a very high spatial resolution magnetogram time sequence from SOT/NFI to study the temporal evolution of spinning and braiding helicities. With a very long time sequence of magnetogram data sets, we found that the helicity injected, due to braiding motion, is larger than the spinning motion even in the presence of a fast rotating emerging sunspot in AR 10930.

Zhang et al. (2007) studied the same active region and found that the $\mathrm{N}$-polarity sunspot rotated about $240^{\circ}$ on its center over a period of three days. In their study, they used the TRACE continuum images and selected a few features to find the rotation rate and the total rotation angle. They found some features rotated at a speed of $10^{\circ}-20^{\circ} \mathrm{hr}^{-1}$. By using the $G$-band images from Hinode/SOT, Min \& Chae (2009) reported that the $\mathrm{N}$-polarity region rotated about its center by $540^{\circ}$. They have also reported that the observed average angular velocity is about $8^{\circ} \mathrm{hr}^{-1}$ and it persisted over a period of two days. By identifying the penumbral fibrils in the continuum images obtained from SP on board Hinode, Yan et al. (2009) estimated the rotation angle and speed. They observed that the penumbral features have rotated over a period of four days by $259^{\circ}$. The rate of rotation was as high as $8^{\circ} \mathrm{hr}^{-1}$ on 2006 December 11 . We used a different data set and method to estimate the rotation rate of the sunspot region P01. We do not use any features to extract the rate of rotation information. We extracted the angular rotation information from the measured spin helicity flux. We observed that region $\mathrm{P} 01$ rotated by about $260^{\circ}$ over a period of three days. The rate of rotation is larger on December 11 at $8^{\circ} \mathrm{hr}^{-1}$ and then it reduced to $4^{\circ} \mathrm{hr}^{-1}$. The whole rate of rotation appeared as damped oscillations. The difference in the rate of rotation measured by different researchers depends on the data and the method used to estimate the rotational velocity. The advantage with our method is that one can estimate the rotational speed for all the partitions without performing a separate individual calculation. It also provides the helicity injected due to the rotation of the region without doing any extra computation.

The regions P01 and N01 had opposite spin until 2006 December 12. During this time of flux emergence, the twist could have propagated along the flux tube from one leg to another through the corona. This could cause the other flux tube to rotate in the opposite direction (Longcope \& Welsch 2000). The propagation of the twist follows the Alfvén speed and is large in the corona. Hence, there is not any observable delay in transferring the twist from one leg to the other. After December 12, the flux tube connecting N01 and P01 could have decoupled and therefore there may not be any coupling of the twist. However, region N01 continues to change its direction of rotation and hence there is a change in the sign of spin helicity flux. 


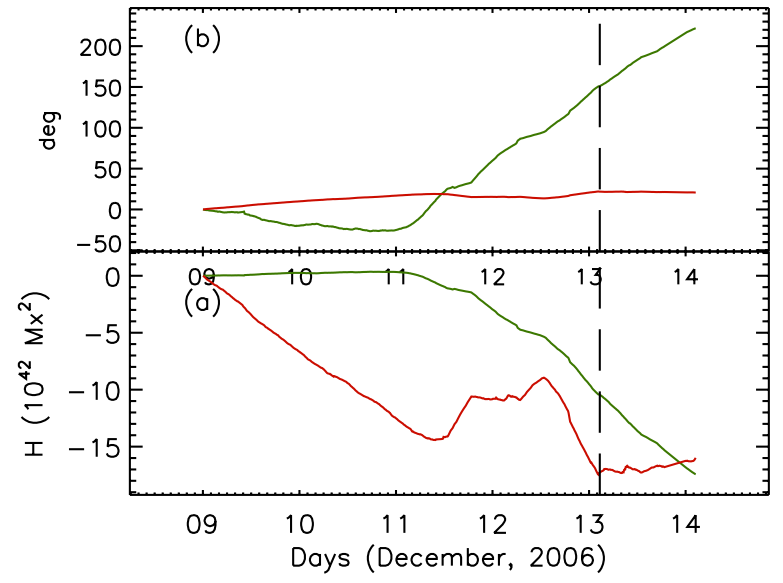

Figure 7. (a) Spin helicity evolution of N01 (red line) and P01 (green line) pole regions is plotted as a function of time. (b) The integrated rotation of the sunspots N01 (red line) and P01 (green line) is plotted as a function of time. The dashed vertical line represents the onset time of the X3.4-class flare. The major and minor tick marks in the $x$-axis represent the days and time of 2006 December.

On December 11, it injected the helicity flux with a positive sign and on December 12, it injected the helicity flux with a negative sign. Just before the X3.4-class flare, it changed its direction of rotation and the sign of spin helicity flux. The change in the sign of the spin helicity flux within the N01 sunspot could have triggered the flare and the CME. This is consistent with the results obtained through the numerical simulation by Kusano et al. (2004). The X3.4-class flare could also be due to other reasons. When the flux tubes are twisted, the current flowing through the flux tubes can produce a Lorentz force, which can attract or repel the magnetic field lines. If the direction of the twist changes suddenly in one of the polarities, then there is a possibility of pushing the oppositely directed field lines toward each other and this could trigger the reconnection. We need to study many such cases to conclude anything conclusively.

The sign of spinning and braiding helicity flux and its magnitude suggests the underneath mechanism of the generation of twist and writhe. Region P01 rotated faster than region N01; however, the spin helicity injected by the N01 region is larger than the $\mathrm{P} 01$ region. This is mainly due to the large magnetic flux of the N01 region. In one of the emerging active regions (AR 8578), Ravindra \& Longcope (2010) found that the spin and braiding helicities are of opposite signs and they interchange their signs during the $50 \mathrm{hr}$ of flux emergence. Unlike that observation, the spinning and braiding helicity in AR 10930 is of the same sign throughout the observation. Even when the spin helicity changed its sign to positive, the braiding helicity followed it. This kind of correlation in the sign of spinning and braiding helicity is found throughout the observations. The direction of rotation in N01 changed about five times during the observations. The initial tilt of the regions is larger than $90^{\circ}$ and deviating from Joy's law tilt. If the counterclockwise rotation of the flux tube corresponds to the negative twist, then the injected spin helicity flux is negative in sign. On the other hand, the clockwise braiding of the flux tubes means positive writhe, which corresponds to the negative braiding helicity (Longcope et al. 2007). In the current situation, the braiding and spinning helicities are of the same sign throughout the observations. Based on the sign of twist and writhe, Nandy (2006) indicated three possibilities for flux tube dynamics. In the present result of the same sign of spinning and braiding helicity fluxes, there could be one possible scenario for the time evolution of braiding

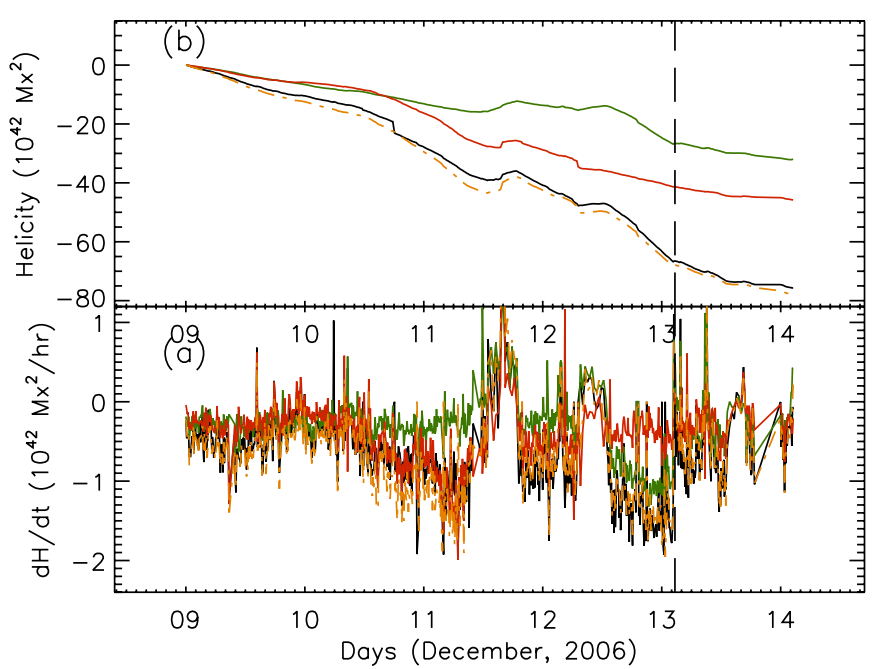

Figure 8. (a) Temporal evolution of the rate of spinning (green), braiding (red), and sum of these two (dash-dotted yellow line) helicity fluxes for all the partitions in the region taken into account. The black curve represents the rate of change of helicity flux estimated independently using Equation (3). (b) Temporal evolution of total accumulated spinning (green), braiding (red), and spin + braiding (dash-dotted) helicities of all the regions taken into account. The black curve represents the total integrated helicity estimated independently. The dark vertical line represents the onset time of the X3.4-class flare. In the $x$-axis, major and minor tick marks represent the days and time of 2006 December.

helicity. When the bipole is emerging, the local rotational motion can spin one or both of the footpoints of an active region. The counterclockwise rotational motion can introduce the negative spinning helicity. Since the helicity is a conserved quantity, this can generate a positive writhe in the tube that corresponds to negative braiding helicity in the active region. In this case, the tilt angle will be away from Joy's law tilt as we saw in the present case. If one changes its sign of helicity (spin helicity) the other (braiding) should also follow it because of the conservation of helicity. The accumulated twist on one leg can propagate into the corona and spin the other footpoint in the opposite direction (Longcope \& Welsch 2000). In AR 10930, the twist could be propagating from the leading polarity (south) to the following polarity (north).

The rate of change of the injected helicity is negative in sign most of the time and it changed its sign five times during the observations. The change in sign of the spin helicity flux is completely dominated by the N01 region. As its spinning direction changed, the sign of the spin helicity flux also changed. The helicity flux density maps $\left(G_{\theta}\right)$ produced by Park et al. (2010) show a similar change in the sign of the helicity flux in the south polarity region, which is large on December 12. In the study of Park et al. (2010) the net rate of helicity flux change occurred five times from December 9-13. We also found at almost similar timings the reversal in the sign of the helicity flux. This sudden change in the sign of helicity could have destabilized the system and hence the large X-class flare could have occurred on December 13 (Kusano et al. 2004).

The change in the sign of the helicity flux was also observed by Park et al. (2010). By using the NLFFF method, Park et al. (2010) reconstructed the coronal magnetic field and estimated the coronal relative magnetic helicity. They observed that during the positive helicity injection there was a decrease in the coronal helicity and hence they concluded that the decrease in helicity flux is due to the helicity carried away by the CME. They also gave another explanation that there is a supply of positive helicity from the photosphere into the corona. We do 
not know the exact reason for the reversal in the sign of the helicity flux. It could be due to the reason explained above. A consequence of opposite helicity injection into the system could lead to the cancellation of the initially injected helicity. In many circumstances, this could lead to reconnection as suggested by Kusano et al. (2004) through their numerical simulations.

The observation of reversal in the sign of the helicity flux has been reported by many earlier researchers, too. Yokoyama et al. (2003) reported the sign reversal of the rate of helicity flux in AR NOAA 8100 during the onset of activity. Nindos et al. (2003) also showed the reversal of the sign of the helicity injection rate during the flare. Moon et al. (2002) found that during the peak time of the flare the helicity flux transported impulsively into the corona. Zhang et al. (2008) observed that there is a sign reversal of helicity flux around the starting time of eruption on 2006 December 13. Park et al. (2010) reported similar results on the same event of 2006 December 13. They also reported that there was a decrease in the coronal relative magnetic helicity one day before the onset of the flare. We have also observed the reversal in the sign of the change in rate of the spin helicity flux during the onset of the X3.4-class flare on 2006 December 13 in line with the others' findings.

In summary, the observed spinning and braiding helicity fluxes possess the same sign in AR 10930 and they maintain their sign correlation throughout the observations. The N-polarity region was emerging and rotating. However, a large contribution to the spinning helicity originates from the S-polarity region. Also, the contribution from the braiding helicity (to the total helicity flux) is larger than the spinning helicity in AR 10930. Finally, there is a reversal in the sign of the spin helicity flux of the S-polarity region many times during its evolution. However, the total accumulated helicity is negative and is about $-7 \times 10^{43} \mathrm{Mx}^{2}$, which is much larger than the helicity flux reported by Zhang et al. (2008). The helicity contained in the flux rope forming the $\mathrm{MC}$ associated with the largest halo $\mathrm{CME}$ observed by LASCO-C 2 on December 13 is also negative and is two orders of magnitude lower than the total injected helicity. The helicity injection of $\sim 1 \mathrm{hr}$ is enough to reach the helicity observed in the cloud. We do not know at this stage whether the sign reversal in spinning and braiding helicity flux is a common phenomenon for all the active regions during its evolution. More such active region studies using high-resolution magnetograms are required in the future.

We thank the referee for fruitful comments which helped us to improve the manuscript. B.R. thanks Dr. Dibyendu Nandi for useful discussions. Hinode is a Japanese mission developed and launched by ISAS/JAXA, with NAOJ as a domestic partner and NASA and STFC (UK) as international partners. It is operated by these agencies in cooperation with ESA and the NSC (Norway). This work was partially supported by Argentinean grants: UBACyT 20020090100264, PIP 11220090100825/10 (CONICET), and PICT-2007-856 (ANPCyT). S.D. is a member of the Carrera del Investigador Cientifico, CONICET. S.D. acknowledges support from the Abdus Salam International Centre for Theoretical Physics (ICTP), as provided in the frame of his regular associateship. This work was funded in part by NASA under contract NNM07AB07C with the Harvard-Smithsonian Astrophysical Observatory.

\section{REFERENCES}

Abramenko, V., Yurchyshyn, V., \& Wang, H. 2008, ApJ, 681, 1669

Barnes, G., Longcope, D. W., \& Leka, K. D. 2005, ApJ, 629, 561
Berger, M. A. 1999, in Geophysical Monograph, Vol. 111, Magnetic Helicity in Space and Laboratory Plasmas, ed. M. R. Brown, R. C. Canfield, \& A. A. Pevtsov (Washington, DC: AGU Press), 1

Brown, D. S., Nightingale, R. W., Alexander, D., et al. 2003, Sol. Phys., 216, 79

Brueckner, G. E., Howard, R. A., Koomen, M. J., et al. 1995, Sol. Phys., 162, 357

Chae, J., Moon, Y.-J., Park, Y.-D., et al. 2007, PASJ, 59, 619

Chae, J., Wang, H., Qiu, J., et al. 2001, ApJ, 560, 476

Dasso, S. 2009, in IAU Symp. 257, Universal Heliophysical Processes, ed. N. Gopalswamy \& D. F. Webb (Cambridge: Cambridge Univ. Press), 379

Dasso, S., Mandrini, C. H., Démoulin, P., \& Farrugia, C. J. 2003, J. Geophys. Res., 108, 1362

Dasso, S., Mandrini, C. H., Démoulin, P., Luoni, M. L., \& Gulisano, A. M. 2005, Adv. Space Res., 35, 711

Dasso, S., Mandrini, C. H., Schmieder, B., et al. 2009, J. Geophys. Res., 114, A02109

Démoulin, P. 2008, Ann. Geophys., 26, 3113

Démoulin, P., \& Berger, M. A. 2003, Sol. Phys., 215, 203

Fan, Y., \& Gibson, S. E. 2004, ApJ, 609, 1123

Guo, Y., Ding, M. D., Wiegelmann, T., \& Li, H. 2008, ApJ, 679, 1629

Hagenaar, H. J. 1999, PhD thesis, Univ. Utrecht

Harra, L. K., Mandrini, C. H., Dasso, S., et al. 2011, Sol. Phys., 268, 213

Ichimoto, K., Lites, B., Elmore, D., et al. 2008, Sol. Phys., 249, 233

Jing, J., Chae, J., \& Wang, H. 2008a, ApJ, 672, L73

Jing, J., Tan, C., Yuan, Y., et al. 2010, ApJ, 713, 440

Jing, J., Wiegelmann, T., Suematsu, Y., Kubo, M., \& Wang, H. 2008b, ApJ, 676, L81

Klein, L. W., \& Burlaga, L. F. 1982, J. Geophys. Res., 87, 613

Kosugi, T., Matsuzaki, K., Sakao, T., et al. 2007, Sol. Phys., 243, 3

Kubo, M., Yokoyama, T., Katsukawa, Y., et al. 2007, PASJ, 59, 779

Kusano, K., Maeshiro, T., Yokoyama, T., \& Sakurai, T. 2004, ApJ, 610, 537

Lepping, R. P., Burlaga, L. F., \& Jones, J. A. 1990, J. Geophys. Res., 95, 11957

Liu, Y., Luhmann, J. G., Müller-Mellin, R., et al. 2008, ApJ, 689, 563

Longcope, D. W., Ravindra, B., \& Barnes, G. 2007, ApJ, 668, 571

Longcope, D. W., \& Welsch, B. T. 2000, ApJ, 545, 1089

Low, B. C. 1994, Phys. Plasmas, 1, 1684

Low, B. C. 1996, Sol. Phys., 167, 217

Lundquist, S. 1950, Ark. Fys., 2, 361

Luoni, M. L., Mandrini, C. H., Dasso, S., van Driel-Gesztelyi, L., \& Démoulin, P. 2005, J. Atmos. Terr. Phys., 67, 1734

Lynch, B. J., Zurbuchen, T. H., Fisk, L. A., \& Antiochos, S. K. 2003, J. Geophys. Res., 108, 1239

Magara, T. 2009, ApJ, 702, 386

Mandrini, C. H., Pohjolainen, S., Dasso, S., et al. 2005, A\&A, 434, 725

Min, S., \& Chae, J. 2009, Sol. Phys., 258, 203

Moon, Y.-J., Chae, J., Wang, H., Choe, G. S., \& Park, Y. D. 2002, ApJ, 580, 528

Nandy, D. 2006, J. Geophys. Res. (Space Physics), 111, A12S01

Nindos, A., \& Zhang, H. 2002, ApJ, 573, L133

Nindos, A., Zhang, J., \& Zhang, H. 2003, ApJ, 594, 1033

November, L. J., \& Simon, G. W. 1988, ApJ, 333, 427

Park, S.-H., Chae, J., Jing, J., Tan, C., \& Wang, H. 2010, ApJ, 720, 1102

Pevtsov, A. A., Maleev, V. M., \& Longcope, D. W. 2003, ApJ, 593, 1217

Ravindra, B. 2006, Sol. Phys., 237, 297

Ravindra, B., \& Howard, T. A. 2010, Bull. Astron. Soc. India, 38, 147

Ravindra, B., \& Longcope, D. W. 2010, in Astrophysics and Space Science Proceedings: Magnetic Coupling between the Interior and Atmosphere of the Sun, ed. S. S. Hasan \& R. J. Rutten (Berlin: Springer), 448

Ravindra, B., Venkatakrishnan, P., \& Kumar, B. 2004, Sol. Phys., 225, 47

Ravindra, B., Venkatakrishnan, P., \& Tiwari, S. K. 2011, Adv. Geosci. Sol. Terres., 27, 153

Scherrer, P. H., Bogart, R. S., Bush, R. I., et al. 1995, Sol. Phys., 162, 129

Schrijver, C. J., De Rosa, M. L., Metcalf, T., et al. 2008, ApJ, 675, 1637

Suematsu, Y., Tsuneta, S., Ichimoto, K., et al. 2008, Sol. Phys., 249, 197

Title, A. M., Tarbell, T. D., Topka, K. P., et al. 1989, ApJ, 336, 475

Tiwari, S. K., Joshi, J., Gosain, S., \& Venkatakrishnan, P. 2008, in Astrophysics and Space Science Proceedings: Turbulence, Dynamos, Accretion Disks, Pulsars and Collective Plasma Processes, ed. S. S. Hasan, R. T. Gangadhara, \& V. Krishan (Berlin: Springer), 329

Tsuneta, S., Ichimoto, K., Katsukawa, Y., et al. 2008, Sol. Phys., 249, 167

Welsch, B. T., \& Longcope, D. W. 2003, ApJ, 588, 620

Yan, X.-L., Qu, Z.-Q., Xu, C.-L., Xue, Z.-K., \& Kong, D.-F. 2009, Res. Astron. Astrophys., 9, 596

Yang, S., Büchner, J., \& Zhang, H. 2009, ApJ, 695, L25

Yokoyama, T., Kusano, K., Maeshiro, T., \& Sakurai, T. 2003, Adv. Space Res., 32, 1949

Zhang, J., Li, L., \& Song, Q. 2007, ApJ, 662, L35

Zhang, Y., Tan, B., \& Yan, Y. 2008, ApJ, 682, L133 